\title{
Characterization of Brassica napus L. genotypes utilizing sequence-related amplified polymorphism and genotyping by sequencing in association with cluster analysis
}

\author{
Corey J. Lees • Genyi Li • Robert W. Duncan
}

Received: 25 July 2016 / Accepted: 20 October 2016/Published online: 10 November 2016

(C) The Author(s) 2016. This article is published with open access at Springerlink.com

\begin{abstract}
Identifying parental combinations that exhibit high heterosis is a constant target for commercial Brassica napus L. hybrid development programs. Finding high heterotic parental combinations can require hundreds of test crosses and years of yield evaluation. Heterotic pool development could be used to divide breeding material into specific breeding pools and focus the number of parental combinations created. Here, we report the genotypic characterization of 79 B. napus genotypes by calculating genetic distance based on sequence-related amplified polymorphism (SRAP) and genotyping by sequencing (GBS) in association with a neighbour-joining clustering algorithm. Despite the different genotypic analyses, neighbour-joining cluster analysis based on genetic distance of SRAP and GBS produced similar clusters. Homology between SRAP and GBS clusters was approximately $77 \%$ when manually comparing clusters and $68 \%$ when comparing clusters using Compare2Trees. This research demonstrates that SRAP can have similar efficacy when compared to next-generation sequencing technology for heterotic pool classification. This information may provide an important breeding scaffold for the development of
\end{abstract}

Electronic supplementary material The online version of this article (doi:10.1007/s11032-016-0576-6) contains supplementary material, which is available to authorized users.

C. J. Lees · G. Li • R. W. Duncan $(\bowtie)$

Faculty of Agricultural and Food Sciences, Department of Plant

Science, University of Manitoba, 222 Agriculture Building,

Winnipeg, MB R3T 2N2, Canada

e-mail: rob.duncan@umanitoba.ca hybrid cultivars based upon genetic distance and cluster analysis.

Keywords GBS · SRAP - Genetic distance $\cdot$ Cluster analysis $\cdot$ Heterosis $\cdot$ Brassica napus

\section{Introduction}

Brassica napus L. (AACC $n=19$ ) has been heralded as one of the most important global oilseed crops, second only to soybean (Glycine max L.) for total annual production (Carré and Pouzet 2014; Wittkop et al. 2009; Lin et al. 2013). This large-scale production is attributed to the improvement of canola quality $(<2 \%$ erucic acid, $<30 \mu \mathrm{mol} / \mathrm{g}$ glucosinolates) and specialty canola cultivars that produce high stability oil for human consumption and meal for animal feed (Rahman 2013). Additionally, high erucic acid rapeseed (HEAR) cultivars also contribute valuable industrial oil for lubricants and slip agents for niche oleochemical markets (Wittkop et al. 2009; McVetty and Duncan 2014). Hybrid B. napus cultivars have replaced their openpollinated counterparts due to the exploitation of heterosis (Rahman 2013). Heterosis occurs when progeny outperform both parents in a variety of agronomic traits but specifically seed yield (Shull 1908; Radoev et al. 2008). Therefore, hybrid cultivars that possess increased yield and superior uniformity in the $\mathrm{F}_{1}$ generation are highly sought after by producers to continually match global demand (Riaz and Quiros 2011). Finding parental combinations that exhibit high heterotic gains may 
require hundreds of test crosses and years of yield evaluation. To alleviate this problem, heterotic pool development has been suggested as a method to separate germplasm and breeding genotypes into distinct clusters for breeding purposes (Rahman 2013; Odong et al. 2011; Girke et al. 2012).

Distinguishing genotypes and the development of heterotic pools can be based on genetic distance and cluster analysis (Ali et al. 1995; Riaz and Quiros 2011; Jesske et al. 2013). Genetic distance is a measure of genetic divergence between species or between individuals and populations within the same species (Nei 1987). Genetic distance can be calculated using a variety of genotypic data sets produced using multiple molecular characterization methods (Jain et al. 1994; Becker et al. 1995; Lombard et al. 2000) along with numerous mathematical formulas (Nei 1972; Cavalli-Sforza and Edwards 1967; Reynolds et al. 1983; Tamura and Nei 1993). Several forms of cluster analysis exist including Ward's method (Ward 1963), unweighted pair group method with arithmetic mean [UPGMA (Sokal and Michener 1958)] and neighbour-joining [NJ (Saitou and Nei 1987)] which are all agglomerative (bottomup) clustering techniques. Applying clustering algorithms to genetic distance models allows for the grouping of genotypes with a short genetic distance together into a heterotic cluster or pool (Odong et al. 2011). Genetic distance and cluster analysis have previously been employed in B. napus hybrid development (Diers et al. 1996; Riaz et al. 2001; Yu et al. 2005). Previous research has shown that $B$. napus parental combinations from different pools or clusters exhibit higher heterosis than those using parents from the same cluster (Ali et al. 1995; Riaz and Quiros 2011). Although clustering techniques have been used for decades in plant breeding, there is no scientific consensus on which clustering method produces the most accurate cluster set for breeding purposes (Mouchet et al. 2008; Odong et al. 2011). Therefore, the molecular characterization method, genetic distance method and clustering method create a large combination of experimental designs that can be used to develop and explore heterotic pools for hybrid development.

Sequence-related amplified polymorphism (SRAP, $\mathrm{Li}$ and Quiros 2001) has been a popular molecular method for the identification of genetic diversity in many crop species including Cucurbita pepo L. (Ferriol et al. 2003), Prunus persica L. (Ahmad et al. 2004) and Lycopersicon esculentum L. (Ruiz et al.
2005). In addition, SRAP has previously been successful in associating genetic distance calculated in the presence/absence of markers with hybrid heterosis based on cluster analysis in B. napus (Riaz et al. 2001; Riaz and Quiros 2011). However, today's nextgeneration sequencing technologies have reduced the cost of DNA sequencing, allowing the ability to evaluate genetic diversity based on single nucleotide polymorphisms (SNPs) (Nielsen et al. 2011; Elshire et al. 2011). Genotyping by sequencing (GBS) is one such next-generation method that can be used for SNP discovery which uses reduced genome representation with methylation-sensitive restriction enzymes (Elshire et al. 2011). Through methylation sensitivity, repetitive regions of the genome can be avoided which simplifies the computational challenge of genome alignment with large, polyploidy genomes (Elshire et al. 2011). To date, several important crop species including corn (Zea mays L.), barley (Hordeum vulgare L.) and rice (Oryza sativa L.) have used GBS for a multitude of downstream applications (Elshire et al. 2011; Liu et al. 2014; Spindel et al. 2013).

The objective of this study was to calculate genetic distance between 79 B. napus genotypes using Nei's standard genetic distance based on SRAP presence/ absence genotyping and the Tamuri-Nei genetic distance formula based on SNPs discovered through GBS. A neighbour-joining clustering method was then used on each separate genetic distance method, and the results were compared. Ultimately, the goal of this research is to improve heterotic pool definitions through multiple techniques and to find a system that will reduce the overall time and space required for discovering high heterotic parental combinations.

\section{Materials and methods}

\section{Brassica napus genotypes}

Seventy-nine B. napus genotypes were selected for this study (Table 1). Most genotypes are considered spring habit germplasm developed for western Canada, with the addition of several European and resynthesized B. napus genotypes. Several genotypes are of canola quality, while the vast majority are considered as HEAR. Of the 79 genotypes, 38 are $o g u$-INRA restorers and 41 are open-pollinated genotypes (maintainers or Blines in the $o g u$-INRA pollination control system). 
Table 1 Seventy-nine spring habit Brassica napus L. genotypes with origin, quality and maintainer or restorer designation for the ogu-INRA pollination control system

\begin{tabular}{|c|c|c|c|c|c|c|c|}
\hline & & & & \multirow{2}{*}{$\begin{array}{c}\text { Red River } \\
1852-\mathrm{B}\end{array}$} & \multirow[b]{2}{*}{$\mathrm{U}$ of $\mathrm{M}$} & \multirow[b]{2}{*}{ HEAR } & \multirow{2}{*}{$\begin{array}{l}\text { restorer } \\
\text { Maintainer }\end{array}$} \\
\hline Identification & Origin & Quality & $\begin{array}{l}\text { Maintainer/ } \\
\text { restorer }\end{array}$ & & & & \\
\hline NEW-620-R & $\mathrm{U}$ of $\mathrm{M}$ & HEAR & \multirow{2}{*}{$\begin{array}{l}\text { Restorer } \\
\text { Restorer }\end{array}$} & \multirow{2}{*}{$\begin{array}{c}\text { Red River } \\
1997-B\end{array}$} & $\mathrm{U}$ of $\mathrm{M}$ & HEAR & \multirow[t]{2}{*}{ Maintainer } \\
\hline NEW-621-R & $\mathrm{U}$ of $\mathrm{M}$ & HEAR & & & \multirow[b]{2}{*}{$\mathrm{U}$ of $\mathrm{M}$} & \multirow[b]{2}{*}{ HEAR } & \\
\hline Castor-R & $\mathrm{U}$ of $\mathrm{M}$ & HEAR & Restorer & $\begin{array}{c}\text { Red River } \\
\text { 1861-B }\end{array}$ & & & Maintainer \\
\hline UMI-71-R & European & HEAR & Restorer & RRHR503-B & $\mathrm{U}$ of $\mathrm{M}$ & HEAR & Maintainer \\
\hline UMS-189-R & European & HEAR & Restorer & RRHR204-B & $\mathrm{U}$ of $\mathrm{M}$ & HEAR & Maintainer \\
\hline 12DH378-R & $\mathrm{U}$ of $\mathrm{M}$ & HEAR & Restorer & RRHR404-B & $\mathrm{U}$ of $\mathrm{M}$ & HEAR & Maintainer \\
\hline 12DH377-R & $\mathrm{U}$ of $\mathrm{M}$ & HEAR & Restorer & RRHR5815-B & $\mathrm{U}$ of $\mathrm{M}$ & HEAR & Maintainer \\
\hline RRHR503-R & $\mathrm{U}$ of $\mathrm{M}$ & HEAR & Restorer & RRHR5819-B & $\mathrm{U}$ of $\mathrm{M}$ & HEAR & Maintainer \\
\hline RRHR204-R & $\mathrm{U}$ of $\mathrm{M}$ & HEAR & Restorer & 08C702-B & $\mathrm{U}$ of $\mathrm{M}$ & HEAR & Maintainer \\
\hline RRHR404-R & $\mathrm{U}$ of $\mathrm{M}$ & HEAR & Restorer & 08C712-B & $\mathrm{U}$ of $\mathrm{M}$ & HEAR & Maintainer \\
\hline RRHR5815-R & $\mathrm{U}$ of $\mathrm{M}$ & HEAR & Restorer & 08C847-B & $\mathrm{U}$ of $\mathrm{M}$ & HEAR & Maintainer \\
\hline RRHR5819-R & $\mathrm{U}$ of $\mathrm{M}$ & HEAR & Restorer & RRHR9707-B & $\mathrm{U}$ of $\mathrm{M}$ & HEAR & Maintainer \\
\hline 08C702-R & $\mathrm{U}$ of $\mathrm{M}$ & HEAR & Restorer & UMI-B & European & HEAR & Maintainer \\
\hline 08C712-R & $\mathrm{U}$ of $\mathrm{M}$ & HEAR & Restorer & UMSA-B & European & HEAR & Maintainer \\
\hline 08C847-R & $\mathrm{U}$ of $\mathrm{M}$ & HEAR & Restorer & UMSH-B & European & HEAR & Maintainer \\
\hline \multirow{2}{*}{$\begin{array}{c}\text { Red River } \\
\text { 1997-R }\end{array}$} & $\mathrm{U}$ of $\mathrm{M}$ & HEAR & Restorer & Venus-B & $\mathrm{U}$ of $\mathrm{M}$ & HEAR & Maintainer \\
\hline & \multirow{2}{*}{$\mathrm{U}$ of $\mathrm{M}$} & \multirow{2}{*}{ HEAR } & Restorer & Neptune-B & $\mathrm{U}$ of $\mathrm{M}$ & HEAR & Maintainer \\
\hline $1826-\mathrm{R}$ & & & & Castor-B & $\mathrm{U}$ of $\mathrm{M}$ & HEAR & Maintainer \\
\hline Red River & $\mathrm{U}$ of $\mathrm{M}$ & HEAR & Restorer & Mill 03-B & $\mathrm{U}$ of $\mathrm{M}$ & HEAR & Maintainer \\
\hline 1852-R & & & & Mercury-B & $\mathrm{U}$ of $\mathrm{M}$ & HEAR & Maintainer \\
\hline $\begin{array}{l}\text { Red River } \\
\text { 1997-R2 }\end{array}$ & U of M & HEAR & Restorer & Hero-B & $\mathrm{U}$ of $\mathrm{M}$ & HEAR & Maintainer \\
\hline UMI-55-R & European & HEAR & Restorer & 08C344-B & $\mathrm{U}$ of $\mathrm{M}$ & HEAR & Maintainer \\
\hline UMI99-R & European & HEAR & Restorer & Reston-B & $\mathrm{U}$ of $\mathrm{M}$ & HEAR & Maintainer \\
\hline 12DH384-R & $\mathrm{U}$ of $\mathrm{M}$ & HEAR & Restorer & 79R713-B & $\mathrm{U}$ of $\mathrm{M}$ & HEAR & Maintainer \\
\hline 12DH430-R & $\mathrm{U}$ of $\mathrm{M}$ & HEAR & Restorer & 79R714-B & $\mathrm{U}$ of $\mathrm{M}$ & HEAR & Maintainer \\
\hline 12DH478-R & $\mathrm{U}$ of $\mathrm{M}$ & HEAR & Restorer & 79R728-B & $\mathrm{U}$ of $\mathrm{M}$ & HEAR & Maintainer \\
\hline 12DH915-R & $\mathrm{U}$ of $\mathrm{M}$ & HEAR & Restorer & 79R729-B & $\mathrm{U}$ of $\mathrm{M}$ & HEAR & Maintainer \\
\hline 12DH949-R & $\mathrm{U}$ of $\mathrm{M}$ & HEAR & Restorer & ER2-B & Resynthesized & HEAR & Maintainer \\
\hline 11DH91-R & $\mathrm{U}$ of $\mathrm{M}$ & HEAR & Restorer & ER3-B & Resynthesized & HEAR & Maintainer \\
\hline 11DH92-R & $\mathrm{U}$ of $\mathrm{M}$ & HEAR & Restorer & ER7-B & Resynthesized & HEAR & Maintainer \\
\hline 11DH97-R & $\mathrm{U}$ of $\mathrm{M}$ & HEAR & Restorer & ER22-B & Resynthesized & HEAR & Maintainer \\
\hline 11DH108-R & $\mathrm{U}$ of $\mathrm{M}$ & HEAR & Restorer & ZSDH2602-B & Resynthesized & HEAR & Maintainer \\
\hline 11DH109-R & $\mathrm{U}$ of $\mathrm{M}$ & HEAR & Restorer & Topas-B & $\mathrm{U}$ of $\mathrm{M}$ & Canola & Maintainer \\
\hline 11DH114-R & $\mathrm{U}$ of $\mathrm{M}$ & HEAR & Restorer & Polo-B & $\mathrm{U}$ of $\mathrm{M}$ & Canola & Maintainer \\
\hline 11DH122-R & $\mathrm{U}$ of $\mathrm{M}$ & HEAR & Restorer & Sentry-B & $\mathrm{U}$ of $\mathrm{M}$ & Canola & Maintainer \\
\hline 11DH137-R & $\mathrm{U}$ of $\mathrm{M}$ & HEAR & Restorer & Global-B & $\mathrm{U}$ of $\mathrm{M}$ & Canola & Maintainer \\
\hline 11DH144-R & $\mathrm{U}$ of $\mathrm{M}$ & HEAR & Restorer & Westar-B & $\mathrm{U}$ of $\mathrm{M}$ & Canola & Maintainer \\
\hline 11DH148-R & $\mathrm{U}$ of $\mathrm{M}$ & HEAR & Restorer & Apollo-B & $\mathrm{U}$ of $\mathrm{M}$ & Low linolenic & Maintainer \\
\hline 11DH149-R & $\mathrm{U}$ of $\mathrm{M}$ & HEAR & Restorer & Stellar-B & $\mathrm{U}$ of $\mathrm{M}$ & Low linolenic & Maintainer \\
\hline 11DH162-R & $\mathrm{U}$ of $\mathrm{M}$ & HEAR & Restorer & 02R276-B & $\mathrm{U}$ of $\mathrm{M}$ & High oleic low & Maintainer \\
\hline Red River & $\mathrm{U}$ of $\mathrm{M}$ & HEAR & Maintainer & & & movicric & \\
\hline 1826-B & & & & & & & cid rapeseed \\
\hline
\end{tabular}

Table 1 (continued)

Identification Origin Quality

Maintainer/

.


Greenhouse production

Initially, three replicates for each genotype were planted at a depth of $1 \mathrm{~cm}$ into plastic $4 \times 3$-well cell packs $(13 \times 13 \times 5 \mathrm{~cm})$ containing Sunshine Metro Mix potting soil (Sungro® Horticulture, MA, USA) from March 5-12, 2014. Plants were grown in a growth chamber (temperature day $22{ }^{\circ} \mathrm{C}$, night $18{ }^{\circ} \mathrm{C}$; light cycle $16 \mathrm{~h}$ light, $8 \mathrm{~h}$ dark) and watered daily. At the two-leaf stage [14 days after planting (DAP)], each plant was transferred to a plastic grower pot $(14.5 \times 15 \mathrm{~cm})$ using Sunshine Metro Mix potting soil. Pots were transferred to an Argus-controlled greenhouse (Argus Control Systems Ltd., Surrey, BC, Canada) with the following specifications (temperature high $25^{\circ} \mathrm{C}$, low $22{ }^{\circ} \mathrm{C}$; relative humidity 40-50\%; light cycle $16 \mathrm{~h}$ light, $8 \mathrm{~h}$ dark) and watered daily. Fertilizer was applied twice, once at the time of transplanting (14 DAP) and once during flowering (50 DAP) using Plant-Prod ${ }^{\circledR}$ water soluble fertilizer (10-52-10) at a concentration of $15 \mathrm{~g} /$ 3.78 1. Insect populations were controlled with Intercept $^{\mathrm{TM}} 60$ WP (imidacloprid, Bayer Environmental Science, Research Triangle Park, NC, USA) added several days after transplant (20 DAP) with a concentration of $4.1 \mathrm{~g} / 1000$ seedlings with 151 of solution per $100 \mathrm{~m}^{2}$ of seeding trays. All mixing and application procedures were followed as per the manufacturer's instructions.

\section{DNA extraction}

For the SRAP genotyping method, DNA was extracted from fresh leaves in April of 2014 by the cetyltrimethylammonium bromide (CTAB) method (Doyle and Doyle 1990) with minor modifications. A 500-600 mg leaf sample was crushed using a mortar and pestle with liquid nitrogen. The ground leaf tissue was added to a $15-\mathrm{ml}$ centrifuge tube. Six milliliters of preheated extraction buffer $(500 \mathrm{ml}$ of $2 \%$ CTAB, 100 mM Tris-HCl, pH 8.0, 1.4 M NaCl, 20 mM EDTA) was then added, and the tube and was vortexed and incubated for $90 \mathrm{~min}$ in a $65^{\circ} \mathrm{C}$ water bath. The tube was cooled to room temperature, and $7 \mathrm{ml}$ of chloroform-isoamyl alcohol (24:1) was added. The solution was mixed gently for $10 \mathrm{~min}$ and centrifuged at 4600 RPM for $16 \mathrm{~min}$. The supernatant was transferred to a new $15-\mathrm{ml}$ tube; 0.5 volumes of 2-propanol were added and mixed gently to precipitate the DNA. The mixture was centrifuged at 4600 RPM for $5 \mathrm{~min}$; the supernatant was removed, and the DNA pellet was washed with $8 \mathrm{ml} 70 \%(v / v)$ ethanol. The pellet was then air-dried and resuspended in $3 \mathrm{ml}$ of doubledistilled water.

Due to purity requirements for GBS, a Qiagen $\AA$ DNeasy Plant Mini Kit (Qiagen, Valencia, CA, USA) was utilized to ensure ultra-pure DNA. All procedures were followed as per manufacturer's instructions for fresh tissue DNA extraction and purification with the addition of 10-min total elusion time $(2 \times 5 \mathrm{~min})$.

\section{DNA concentration}

DNA quantity for SRAP was determined using a Thermo Scientific NanoDrop 2000 spectrophotometer in conjunction with software NanoDrop 2000 (Thermo Fisher Scientific Inc., MA, USA) with all protocols followed as per manufacturer's instructions for doublestranded DNA quantification. All samples achieved a minimum DNA concentration of $30 \mathrm{ng} / \mu \mathrm{l}$ with a 260 / 280 ratio of $\geq 1.8$. All DNA concentrations for GBS were quantified using a Life Technologies Qubit® Fluorometer (Life Technologies, Carlsbad, CA, USA) with all protocols followed as per manufacturer's instructions for double-stranded DNA quantification. All samples reached a minimum concentration of $50 \mathrm{ng} / \mu \mathrm{l}$ as per GBS requirements (http://www.biotech.cornell. edu/brc/genomic-diversity-facility).

Sequence-related amplified polymorphism

Sequence-related amplified polymorphism is a polymerase chain reaction (PCR) method, which is designed to amplify open reading frames (ORFs) using a variable forward and reverse primer system (Li and Quiros 2001). Each primer is $17-18$ base pairs long with the forward primer containing a core sequence of CCGG. This forward core sequence targets ORFs due to the known distribution that exons are $\mathrm{GC}$ rich $(\mathrm{Li}$ and Quiros 2001). The reverse primer has a core sequence of AATT near the $3^{\prime}$ region to target introns and promoter regions that are typically AT rich ( $\mathrm{Li}$ and Quiros 2001). Together, these primer combinations create polymorphic DNA bands that are separated through electrophoresis in polyacrylamide gels and visualized through autoradiography ( $\mathrm{Li}$ and Quiros 2001). The presence/ absence scoring is then applied to the visualized polymorphic bands, and genotypes can be separated based on scoring (Li and Quiros 2001). 
Primer design followed the protocol of Li and Quiros (2001). Supplemental Table 1 displays the base pair sequence of all primers used in this research. Primer combinations consisted of 29 forward and reverse primer sets used to genotype 79 B. napus genotypes (Supplemental Table 2).

\section{Polymerase chain reaction}

Ten microlitre aliquots of PCR master mix were allocated into a 384-well plate. The master mix was composed

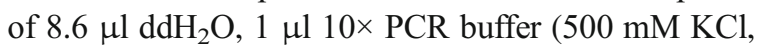
$100 \mathrm{mM}$ Tris, $1 \%$ Triton, $1.5 \mathrm{mM} \mathrm{MgCl}_{2}, \mathrm{pH}$ 9.3), $0.15 \mu \mathrm{l}$ dNTPs, $0.15 \mu \mathrm{l}$ forward primer (Table 2), $0.15 \mu$ l reverse primer (Table 2) and $0.15 \mu \mathrm{l} \mathrm{Taq}$ polymerase. DNA was transferred via a stainless steel 96 spike stamping plate and sealed with a PCR plate cover for the PCR procedure. PCR was completed using the following parameters: temperature cycle initiated at $94{ }^{\circ} \mathrm{C}$ for $3 \mathrm{~min}$ with cycle 2 at $94{ }^{\circ} \mathrm{C}$ for $55 \mathrm{~s}$, cycle 3 was $35{ }^{\circ} \mathrm{C}$ for $55 \mathrm{~s}$ and cycle 4 was $72{ }^{\circ} \mathrm{C}$ for $55 \mathrm{~s}$. Cycles 2-4 were repeated five times. Cycle 5 was set to $94{ }^{\circ} \mathrm{C}$ for $55 \mathrm{~s}$; cycle 6 was $50{ }^{\circ} \mathrm{C}$ for $55 \mathrm{~s}$, and cycle 7 was set to $72{ }^{\circ} \mathrm{C}$ for $55 \mathrm{~s}$. Cycles 5-7 were repeated 30 times. After the final cycle was completed, the PCR products were separated by denaturing acrylamide gels and detected by autoradiography with an ABI Prism 3130XL in association with GenScan ${ }^{\circledR}$ software (V.3.7) (Applied Biosystems, Life Technologies, Carlsbad, CA, USA) (Li and Quiros 2001).

Genotyping by sequencing

GBS is a method for measuring SNPs and utilizes the Illumina ${ }^{\circledR}$ next-generation sequencing technology (Elshire et al. 2011). GBS is a highly multiplexed PCR method that uses a reduced representation of genome complexity through the use of restriction enzymes (REs) that are methylation sensitive (Elshire et al. 2011). This greatly simplifies sequencing and alignment procedures allowing for deep coverage in gene-rich regions (Chen et al. 2013; Elshire et al. 2011). Following RE digestion, adapter barcodes are ligated to the RE cut site allowing many samples to be pooled into one Illumina flow cell greatly reducing cost (Elshire et al. 2011; Chen et al. 2013). Generally, millions of sequence tags (64 bp reads) are generated and 10,000 s to 100,000 s of SNPs can be called with a very high degree of accuracy through a novel GBS computational pipeline, Tassel (Bradbury et al. 2007; Elshire et al. 2011; Glaubitz et al. 2014).

One 96-well plate (Eppendorf twintec PCR plate 96 well) (caps: Thermo Scientific PCR 8 Strip Flat Caps) with DNA samples of 95 B. napus genotypes was submitted to Cornell University Institute of Biotechnology (http://www.biotech.cornell.edu/brc/genomicdiversity-facility) where GBS was completed as per the protocol defined by Elshire et al. (2011). ApeKI (GCWGC, where $\mathrm{W}$ is $\mathrm{A}$ or $\mathrm{T}$ ) was the restriction enzyme chosen at a 95-plex level. All bioinformatics analysis (SNP calling) was completed by Cornell University Institute of Biotechnology using the Tassel computation pipeline V. 3.0.166 (Bradbury et al. 2007; Glaubitz et al. 2014) and the B. napus reference genome (Chalhoub et al. 2014). Genome alignment was generated with Burrows-Wheeler transform algorithm (BWA) version 0.7.8-r455 ( $\mathrm{Li}$ and Durbin 2010; Li and Homer 2010).

\section{Cluster analysis}

For SRAP, each presence/absence marker was scored using a binary system of 1 for present and 0 for absent creating a matrix. Genetic distance using Nei's standard

Table 2 Total number of sequence reads, good barcoded reads and resulting tags for each sequence run of genotyping by sequencing on 95 Brassica napus genotypes

\begin{tabular}{lllll}
\hline & $\begin{array}{l}\text { Barcodes } \\
\text { found in lane }\end{array}$ & $\begin{array}{l}\text { Total number of } \\
\text { reads per lane }\end{array}$ & $\begin{array}{l}\text { Total number of good } \\
\text { barcoded reads }\end{array}$ & $\begin{array}{l}\text { Resulting number } \\
\text { of tags }^{\mathrm{c}}\end{array}$ \\
\hline Run 1 & 96 & $132,278,340$ & $126,177,590$ & $8,110,178$ \\
Run 2 & 96 & $121,989,805$ & $116,807,543$ & $6,580,155$ \\
\hline
\end{tabular}

\footnotetext{
${ }^{a}$ A read is a single sequence generated by the GBS assay

${ }^{\mathrm{b}}$ A good barcoded read was recorded if (1) the read perfectly matched the barcode sequence and the four base remnant ApeK1 cut site, (2) there were no adapter/adapter dimers and (3) contained no $N \mathrm{~s}$ (missing) up to the trim length (Glaubitz et al. 2014)

${ }^{\mathrm{c}} \mathrm{A}$ tag is a unique sequence from the good barcoded reads
} 
genetic distance (Nei 1972) formula was calculated using the software Powermarker (V3.25) (Liu and Muse 2005; http://statgen.ncsu.edu/powermarker/) based upon the binary matrix. This calculation created a new matrix of $79 \times 79$ genotypes with the genetic distance between each genotype displayed. Neighbour-joining cluster analysis (Saitou and Nei 1987) was then applied to the genetic distance matrix using the software Powermarker (V3.25). This created a reference tree with branch lengths between genotypes approximately equal to the genetic distance between genotypes. Tree robustness was tested with the generation of 1000 bootstrapping replicates (Felsenstein 1985). The reference tree file and the 1000 bootstrapping replicate trees were exported as Newick format into Mega 6 (V.6.06 [6140226] Tamura et al. 2013; http://www. megasoftware.net/) and exported as Newick file to be compatible with the software Geneious V.8.05 (Kearse et al. 2012; http://www.geneious.com/download). Consensus tree construction based on the 1000 bootstrapping replicates was completed in Geneious V.8.05 with the following parameters: support threshold set to 0 , topology threshold set to 0 , burn in set to 0 and a greedy clustering model.

For GBS, 80,005 filtered biallelic SNPs were imported into the software program Geneious V.8.05. Only 79 of the 95 genotypes were analysed to match the SRAP analysis. Genetic distance was calculated by Geneious based on the Tamura-Nei distance model (Tamura and Nei 1993), and the subsequent distance matrix was clustered using neighbour-joining method (Saitou and Nei 1987). This created a reference tree with branch lengths between genotypes approximately equal to the genetic distance between genotypes. Tree robustness was tested with 1000 bootstrapping replicates in Geneious V.8.05 with the following parameters: support threshold set to 0 , topology threshold set to 0 , burn in set to 0 and a greedy clustering model.

\section{Cluster similarity}

Cluster similarity was explored on a cluster-by-cluster level, where each conserved cluster $(K)$ was compared to each other for $n$ number of matches, and each match for each cluster was signified as a match percentage for each cluster. This consisted of a branch-to-branch comparison of conserved clusters within each tree. This allows a computation of the similarity of each conserved cluster and the overall similarity between dendrograms using a match percent despite changes in topology. Secondly, cluster similarity was computed using the Java applet Compare2Trees (Nye et al. 2006; http://www.mrc-bsu.cam.ac.uk/personal/thomas/phylo_ comparison/comparison_page.html.). In short, two phylogenetic trees, $T_{1}$ and $T_{2}$, that share the same set of leaves $(L)$ can be compared and can be either rooted or unrooted. The comparison algorithm has two stages: First, every pair of edges $(i, j)$ with $i \in T_{1}$ and $j \in T_{2}$ is assigned a score $s(i, j)$ that reflects the topological similarity of the branches $i$ and $j$. Secondly, branches in the two trees are paired up to optimize the overall score creating a branch-to-branch comparison (Nye et al. 2006).

\section{Results}

Sequence-related amplified polymorphism

Twenty-nine forward and reverse primer combinations amplified through PCR and visualized through autoradiography resulted in 293 polymorphic bands between the 79 B. napus genotypes. On average, each primer combination amplified 10 polymorphic bands per genotype (Supplementary Fig. 1).

\section{Genotyping by sequencing}

The first GBS run produced $\sim 126$ million barcode reads, $84 \%$ of the minimum 150 million barcode reads that Cornell's Institute of Biotechnology has set as their standard. As a result, our GBS material was sequenced a second time generating an additional $\sim 116$ million barcode reads and combined with the first sequencing run (Table 2). This combined pool generated $\sim 8,110,000$ and $\sim 6,580,000$ unique sequence tags, respectively, for a combined total of 1,631,637 filtered sequence tags. Filtered sequence tags are tags at or above a defined threshold for all taxa (samples) in the experiment and were used for genome alignment (Glaubitz et al. 2014). Of those filtered sequence tags, 925,657 (56.7\%) aligned to unique positions, 420,244 $(25.8 \%)$ aligned to multiple positions and 285,736 $(17.5 \%)$ could not be aligned to the reference genome. From the alignment results, all unique aligned filtered sequence tags $(925,657$ or $56.7 \%)$ were used for SNP calling based on the reference genome provided (Chalhoub et al. 2014). The resulting SNPs called from 


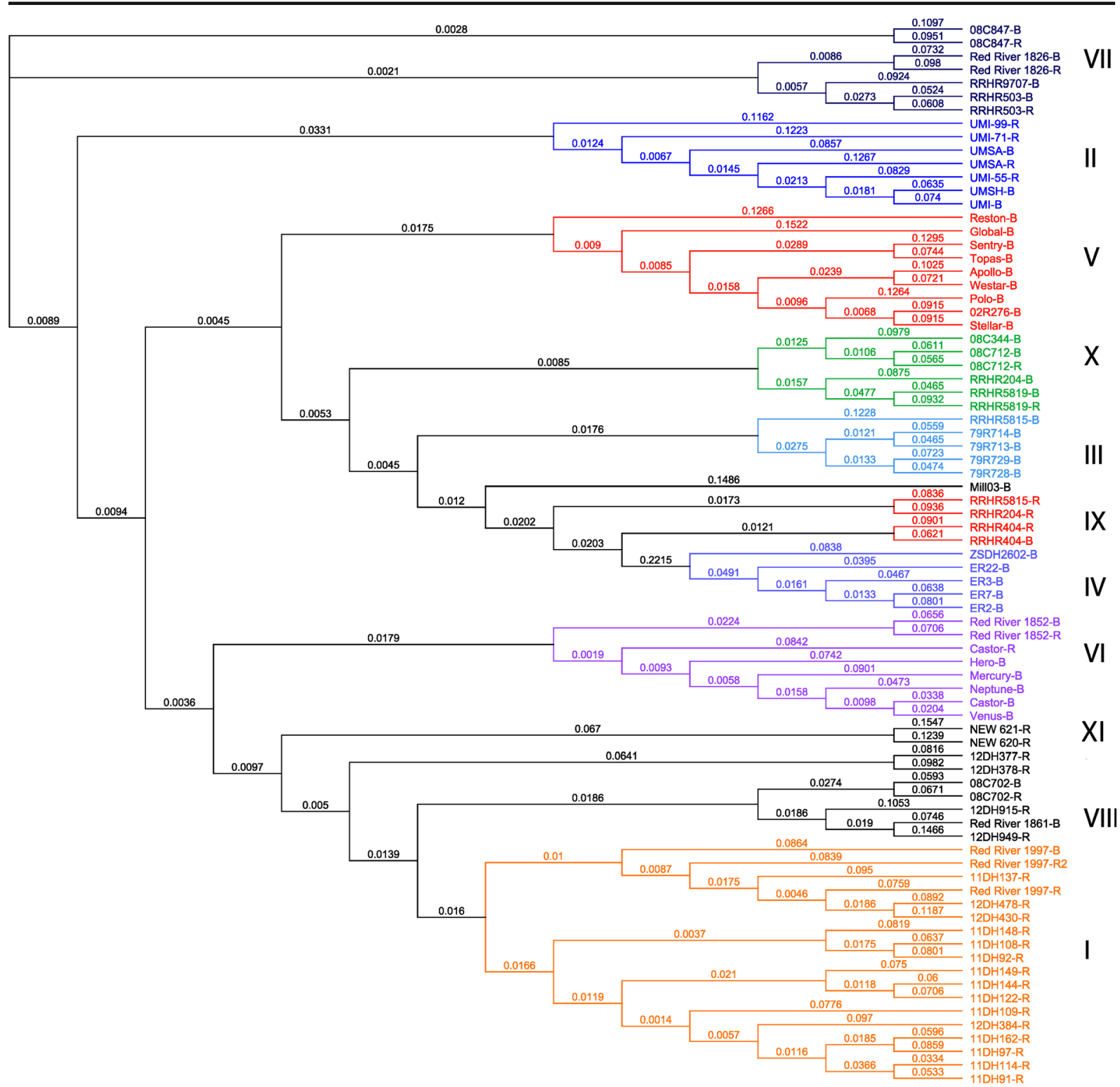

Fig. 1 Neighbour-joining dendrogram clustered using Nei's standard genetic distance based on 293 SRAP polymorphic bands obtained through sequence-related amplified polymorphisms on 79 Brassica napus genotypes visualized in Geneious V.8.05.

the unique aligned positions were divided into three distinct categories [VCF, HapMap (unfiltered), HapMap (filtered)]. The VCF SNPs and HapMap SNPs were called independently, and variation between the two formats was expected. VCF SNPs were called using the algorithm from Catchen et al. (2011) called Stacks, whereas HapMap SNPs were called in Tassel (Bradbury et al. 2007; Glaubitz et al. 2014). Stacks SNP calling resulted in 382,560 VCF SNPs. Tassel SNP calling
Distinct clusters have been numbered and colour-coded for ease of viewing. Each genotype is either a maintainer (-B) or restorer ($\mathrm{R})$ in the $o g u$-INRA pollination control system

generated 179,974 unfiltered SNPs stored in HapMap format. HapMap SNPs were filtered on missingness and allele frequency which generated 80,005 high-quality bi-allelic SNPs.

\section{Cluster analysis}

The neighbour-joining cluster analysis based on the SRAP genetic distance matrix produced a reference 
dendrogram with 11 distinct heterotic clusters (Fig. 1). Dendrogram robustness was tested through 1000 bootstrapping replications (Supplementary Fig. 2). The bootstrapping dendrogram also produced 11 heterotic clusters. However, only five clusters (II, IV, V, VII and IX) remained identical over 1000 replications. These can be considered high confidence heterotic clusters, although only minor genotype movement was observed throughout the other non-identical clusters. The neighbour-joining cluster analysis based on the GBS distance matrix produced a reference dendrogram with 12 heterotic clusters (Fig. 2). Again, tree robustness was tested with 1000 bootstrapping replications (Supplementary Fig. 3). Between the GBS reference tree and the bootstrapping tree (Fig. 2 and Supplemental Fig. 3), only two heterotic clusters (IV and V) did not remain identical over 1000 replications. The GBS bootstrapping tree showed remarkable

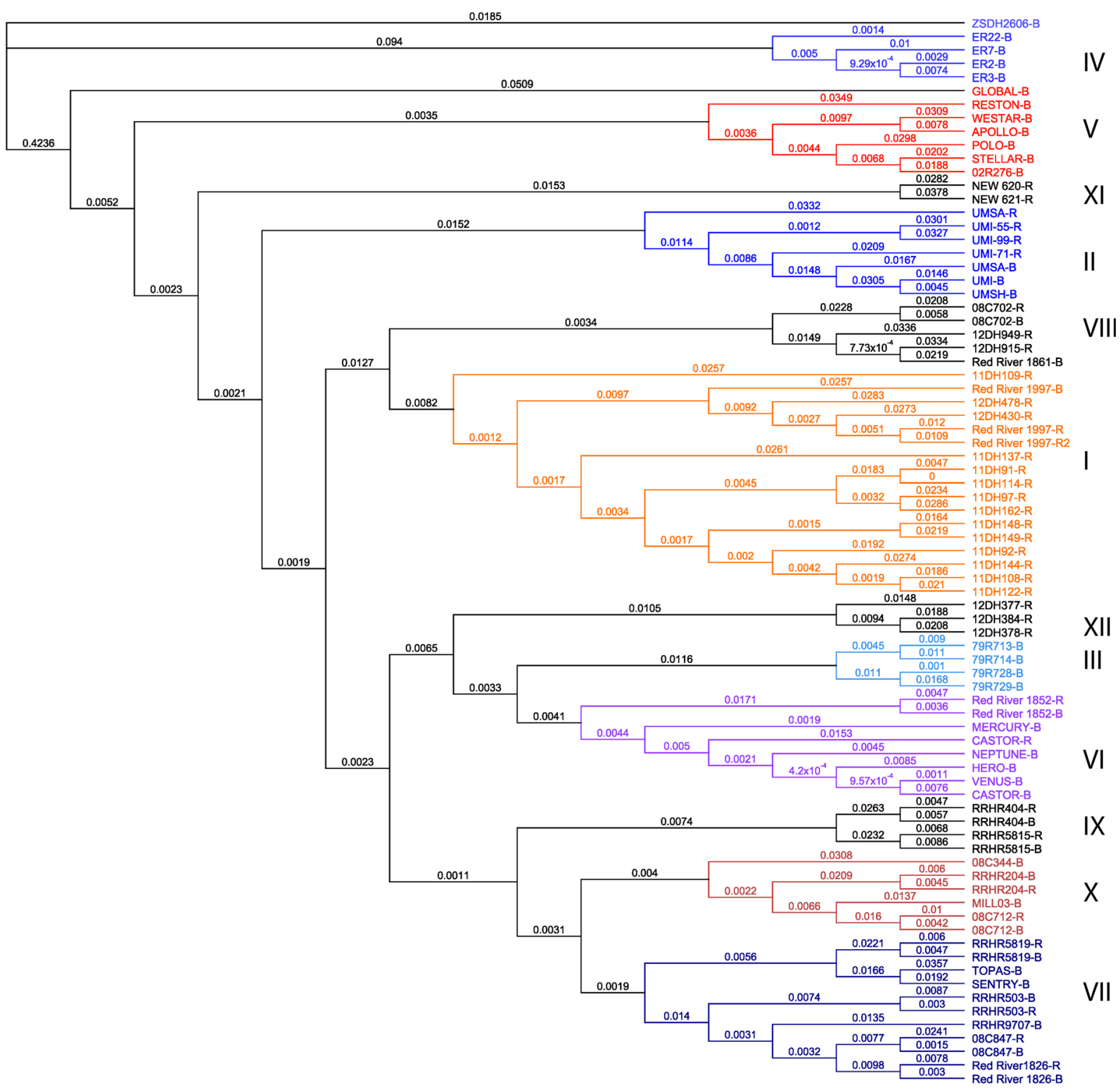

Fig. 2 Neighbour-joining dendogram based on Tamura-Nei's genetic distance calculated on 80,005 SNPs obtained from genotyping by sequencing on 79 Brassica napus genotypes visualized in Geneious (V.8.05). Distinct clusters have been numbered and colour-coded for ease of viewing. Each genotype is either a maintainer (-B) or restorer (-R) in the ogu-INRA pollination control system 
robustness as many nodes have a $90 \%$ or higher clustering percent over 1000 bootstrapping replications (Felsenstein 1985), and considerable confidence can be given to a tree that is supported by $>80 \%$ of bootstrapping replicates (Zharkikh and Li 1992). On the other hand, little confidence can be given to a tree that is supported by $<75 \%$ of the replicates (Zharkikh and Li 1992). This may apply to the SRAP bootstrapping tree as it seemed to vary over 1000 replications, and its node length ranged from approximately 1.3 to $99.5 \%$ intervals. Comparing all dendrograms, cluster II remained identical through the different methods and replicates. Cluster II is represented by genotypes of European descent.

Genetic distance

SRAP genetic distance based on Nei's genetic distance formula calculated on 293 polymorphic bands had a genetic distance range of 0.08 between genotypes 11DH91-R and 11DH114-R to 0.74 between genotypes ER2-B and NEW-620-R (Fig. 1). GBS genetic distance was calculated using the Tamura-Nei formula using 80,005 SNPs, which had a range of 0.0047 between genotypes $11 \mathrm{DH} 91-\mathrm{R}$ and $11 \mathrm{DH} 114-\mathrm{R}$ to 0.629 between genotypes ER3-B and 08C847-R (Fig. 2).

\section{Cluster similarity}

Cluster similarity was investigated between the two genotypic methods of SRAP and GBS in association with Nei's standard genetic distance and the TamuraNei distance model, respectively. Cluster topology differed between the genetic distance calculated on Nei's standard genetic distance based on SRAP and the genetic distance calculated on the Tamura-Nei model based on GBS despite using the same neighbourjoining algorithm (Table 3). However, distinct clusters contained similar genotypes between the two methods. Clusters II, IV, VI and XI are all examples where all genotypes remained identical over both methods.

Despite the differences in cluster placement, each cluster between the two dendrograms shows highly conserved clusters for specific genotypes (Figs. 1 and 2). There is an approximate homology of $77 \%$ between all genotypes in all clusters when manually compared. The Java applet Compare2Trees was implemented for a branch-to-branch computational comparison (Nye et al.
Table 3 Manual comparison of similarity for each conserved cluster between the genetic distances of sequence-related amplified polymorphism and genotyping-by-sequencing dendrograms for individual genotypes $(n)$

\begin{tabular}{lrcc}
\hline $\begin{array}{l}\text { Conserved clusters between } \\
\text { dendrograms }\end{array}$ & SRAP $(n)^{\mathrm{a}}$ & GBS $(n)^{\mathrm{b}}$ & Match $^{\mathrm{c}}$ \\
\hline I & 18 & 17 & \\
II & 7 & 7 & 100 \\
III & 5 & 4 & 80 \\
IV & 5 & 5 & 100 \\
V & 9 & 7 & 78 \\
VI & 8 & 8 & 100 \\
VII & 7 & 11 & 64 \\
VIII & 7 & 5 & 71 \\
IX & 5 & 4 & 60 \\
X & 6 & 6 & 71 \\
XI & 2 & 2 & 100 \\
XII & 0 & 3 & 0 \\
Total $(n)$ and mean match & 79 & 79 & 76.5 \\
\multicolumn{1}{c}{ percent $(\%)$} & & & \\
\hline
\end{tabular}

${ }^{\mathrm{a}}$ The total number of genotypes separated by neighbour-joining cluster analysis based on SRAP genetic distance

${ }^{b}$ The total number of genotypes separated by neighbour-joining cluster analysis based on GBS genetic distance

${ }^{\mathrm{c}}$ Match percent obtained by dividing the smaller number of genotypes per cluster by the larger number of genotypes per cluster

2006). Compare2Trees found an approximate homology of $68 \%$ between the SRAP and GBS trees.

\section{Discussion}

Several primer combinations for SRAP have been previously been reported and found to be successful for differentiating B. napus genotypes ( $\mathrm{Li}$ and Quiros 2001; Sun et al. 2007; Wen et al. 2006; Riaz et al. 2001). Sun et al. (2007) constructed an ultra-dense genetic map using 1634 SRAP primer combinations to produce 13,551 mapped markers. Wen et al. (2006) discovered 123 polymorphic fragments using 25 SRAP primer combinations, and Riaz et al. (2001) found 118 polymorphic bands based on 18 forward and reverse SRAP primer combinations. Here, we report 293 polymorphic bands with 29 forward and reverse primer combinations for 79 B. napus genotypes.

Genotyping by sequencing is a relatively new protocol for high-throughput SNP detection (Elshire et al. 2011). There is currently little GBS data published for 
B. napus diversity. We report that 285,736 tags or $17.5 \%$ of filtered tags could not be aligned to the B. napus reference genome (Chalhoub et al. 2014). Elshire et al. (2011) reported that only $2 \%$ of parental maize line B73 filtered tags could not be aligned to the maize reference genome (B73 RefGen V.1). Elshire et al. (2011) found that this $2 \%$ of non-aligning reads were not present in the reference genome. In the current research, $17.5 \%$ could not be aligned, and in conjunction with the Elshire et al. (2011) findings, these sequencing tags are probably not contained within the reference genome. Currently, the B. napus genome is of winter habit and is an open-pollinated genotype (Chalhoub et al. 2014). This may explain a vast majority of non-aligning reads as our material is considered to be spring habit and 38 of the 79 genotypes contain restorer introgressions from Raphanus sativa L. for use in the ogu-INRA pollination control system (Heyn 1976; Delourme and Eber 1992; Gourret et al. 1992; Delourme et al. 1994). These two differences may contribute to the unaligned sequences; however, this hypothesis warrants further investigation.

Genetic distance has been a well-cited mathematical tool for the determination of species and/or individual relatedness (Ali et al. 1995; Riaz et al. 2001; Yu et al. 2005; Jesske et al. 2013). However, very few studies present multiple genetic distance methods with the same population or genotypes with the same clustering method for the purpose of evaluating genetic distance measures. Here, the ultimate goal is to investigate which genetic distance method can produce highly accurate heterotic pools for the purpose of predicting heterosis. To pursue this endeavour, intra-cluster and inter-cluster hybrids from the current dendrograms need to be evaluated to gauge which genetic distance method has greater predictive power. However, despite these different methods, genotypes 11DH91-R and 11DH114-R produced the smallest genetic distance using both methods. In addition, the largest values obtained for both genetic distance methods involved Canadian $B$. napus genotypes compared to resynthesized B. napus, and this is in agreement with Jesske et al. (2013) who presented evidence that resynthesized B. napus genotypes contain genetic diversity not seen in elite breeding lines. This experimental evidence lends credibility to both SRAP and GBS methods as each separate mathematical formula calculated the shortest genetic distance between the same pair of genotypes and also produced the largest genetic distance between Canadian B. napus genotypes and resynthesized genotypes.

The comparison between the genetic distance dendrograms derived from SRAP and GBS (Figs. 1 and 2) was remarkably similar despite using different genetic distance formulas and different genotypic methods (77\% homology based on manual match percent and $68 \%$ homology calculated by the Java applet Compare2Trees). The close association in percentage shows that these trees are similar; however, when bootstrapping values were incorporated, the GBS bootstrapping tree was stronger and more robust as opposed to the SRAP bootstrapping dendrogram (Supplemental Figs. 2 and 3). Unfortunately, the program Compare2Trees cannot compare bootstrapping trees (Nye et al. 2006). However, across all dendrograms, a visual inspection shows that cluster II remained identical. This supports the conclusions of Diers et al. (1996) and Cuthbert et al. (2009). Specifically, Cuthbert et al. (2009) showed that European-derived breeding material was distinct from Canadian high erucic acid rapeseed material based on heterotic performance and cluster II in this analysis retains only European-derived genotypes.

From a B. napus breeding standpoint, it is well cited that inter-cluster hybrids exhibit higher heterosis than intra-cluster hybrids (Grant and Beversdorf 1985; Riaz et al. 2001; Riaz and Qiuros 2011). This assumption is also well documented in maize hybrid breeding as many commercial hybrids are from complimentary heterotic pools (e.g. Reid Yellow Dent and Lancaster Sure Crop) (Lu et al. 2009; Chen et al. 2015). The conserved clusters between SRAP and GBS (II, IV, VI and XI) suggest that the genotypes within each cluster may be more important for heterotic gains than cluster placement in the overall topology of the dendrogram, since topologies shift between methods. Genotypic placement within clusters can be considered highly accurate given origin and pedigree information. For instance, cluster I for both methods contains mostly double haploid (DH) material and Red River 1997, a parental genotype for most of the DH material. Cluster II was all European-sourced material; cluster IV was all resynthesized genotypes, and cluster VI contains genotypes released by the University of Manitoba (Scarth et al. 1991; Scarth et al. 1995; McVetty et al. 1996a,b; McVetty et al. 1998; McVetty et al. 2006). 
Further investigation between inter-cluster and intracluster hybrids as well as cluster placement and the genetic distance between each parental genotype would prove extremely useful for developing a breeding schematic based on genetic distance and cluster analysis for B. napus hybrids. Since cluster II was conserved across all methods and replicates, this European-derived cluster is distinct and future inter-cluster hybrids should be explored using this cluster.

From a monetary standpoint, GBS was outsourced to Cornell University Institute of Biotechnology which (as of 2014) roughly had a price tag of US\$38.00 per sample for one 96-well plate including bioinformatics with an addition cost of approximately US $\$ 4.50$ per sample for DNA extraction using a Qiagen ${ }^{\circledR}$ DNeasy Plant Mini Kit extraction kit and an additional $\$ 0.93$ per sample for labour. In relation, CTAB costs US $\$ 0.80$ per genotype for DNA extraction, assessed by the Abarshi et al. (2010). CTAB is more laborious and takes longer giving it a higher labour cost per sample at US\$1.25. However, PCR reagent costs were assessed by Duncan et al. (2012) at US $\$ 1.22$ for a total cost of US $\$ 3.27$ per sample for SRAP opposed to US\$43.49 for GBS. Comparing the overall resolution between the two methods at $\sim 77$ and $68 \%$ similarity, respectively, SRAP appears to be as effective for separating diverse genotypes into distinct clusters for breeding purposes with a substantially lower cost. Despite the monetary difference, the similarity in clustered genotypes between each method lends credibility to both methods. Since these methods are roughly a decade apart, yet still produce similar results, we can infer that these genotypic methods are comparable when investigating heterotic pool placement based on cluster analysis and genetic distance in $B$. napus genotypes.

These current heterotic clusters as defined by SRAP and GBS may prove useful for the development of hybrid B. napus cultivars based on genetic distance. Future investigations need to concentrate on the accuracy of genotypic placement through inter-cluster and intra-cluster hybrids with the concurrent measure of hybrid heterosis over parental values to gauge the degree that genetic distance influences heterotic gain.

Acknowledgments The authors acknowledge Ralph Kowatsch and Duoduo Wang for their research contribution and the National Science and Engineering Research Council of Canada, Bunge Canada, DL Seeds and the University of Manitoba for their funding support.

\section{Compliance with ethical standards}

Conflict of interest The authors declare that they have no conflict of interest.

Open Access This article is distributed under the terms of the Creative Commons Attribution 4.0 International License (http:// creativecommons.org/licenses/by/4.0/), which permits unrestricted use, distribution, and reproduction in any medium, provided you give appropriate credit to the original author(s) and the source, provide a link to the Creative Commons license, and indicate if changes were made.

\section{References}

Abarshi MM, Mohammed IU, Wasswa P, Hillocks RJ, Holt J, Legg JP, Seal SE, Maruthi MN (2010) Optimization of diagnostic RT-PCR protocols and sampling procedures for the reliable and cost-effective detection of cassava brown streak virus. J Virol Methods 163(2):353-359

Ahmad R, Potter D, Southwick SM (2004) Genotyping of peach and nectarine cultivars with SSR and SRAP molecular markers. J Am Soc Hortic Sci 129(2):204-210

Ali M, Copeland LO, Elias SG, Kelly JD (1995) Relationship between genetic distance and heterosis for yield and morphological traits in winter canola (Brassica napus L.). Theor Appl Genet 91(1):118-121

Becker HC, Engqvist GM, Karlsson B (1995) Comparison of rapeseed cultivars and resynthesized lines based on allozyme and RFLP markers. Theor Appl Genet 91(1):62-67

Bradbury PJ, Zhang Z, Kroon DE, Casstevens TM, Ramdoss Y, Buckler ES (2007) TASSEL: software for association mapping of complex traits in diverse samples. Bioinformatics 23(19):2633-2635

Carré P, Pouzet A (2014) Rapeseed market, worldwide and in Europe. OCL 21(1):D102

Catchen JM, Amores A, Hohenlohe P, Cresko W, Postlethwait JH (2011) Stacks: building and genotyping loci de novo from short-read sequences. G3: Genes, Genom Genet 1(3):171-182

Cavalli-Sforza LL, Edwards AW (1967) Phylogenetic analysis. Models and estimation procedures. Am J Hum Genet 19(3):233

Chalhoub B, Denoeud F, Liu S et al (2014) Early allopolyploid evolution in the post-Neolithic Brassica napus oilseed genome. Science 345(6199):950-953

Chen C, Mitchell SE, Elshire RJ, Buckler ES, El-Kassaby YA (2013) Mining conifers' mega-genome using rapid and efficient multiplexed high-throughput genotyping-bysequencing (GBS) SNP discovery platform. Tree Genet Genomes 9(6):1537-1544

Chen HM, Zhang YD, Jiang FY, Huang YX, Yao WH, Chen XH, Fan XM (2015) Genetic and heterosis analyses using Hayman's six-generation model for grain yield and yield components in maize. Crop Sci 55(3):1006-1016

Cuthbert RD, Crow G, McVetty PBE (2009) Assessment of agronomic performance and heterosis for agronomic traits in hybrid high erucic acid rapeseed (HEAR). Can J Plant Sci 89(2):227-237 
Delourme R, Eber F (1992) Linkage between an isozyme marker and a restorer gene in radish cytoplasmic male sterility of rapeseed (Brassica napus L.). Theor Appl Genet 85(2-3): $222-228$

Delourme R, Bouchereau A, Hubert N, Renard M, Landry BS (1994) Identification of RAPD markers linked to a fertility restorer gene for the Ogura radish cytoplasmic male sterility of rapeseed (Brassica napus L.). Theor Appl Genet 88(6-7): 741-748

Diers BW, McVetty PBE, Osborn TC (1996) Relationship between heterosis and genetic distance based on restriction fragment length polymorphism markers in oilseed rape (Brassica napus L.). Crop Sci 36(1):79-83

Doyle JJ, Doyle JL (1990) Isolation of DNA from small amounts of plant tissues. BRL focus 12:13-15

Duncan RW, Gilbertson RL, Singh SP (2012) Direct and markerassisted selection for resistance to common bacterial blight in common bean. Crop Sci 52(4):1511-1521

Elshire RJ, Glaubitz JC, Sun Q, Poland JA, Kawamoto K, Buckler ES, Mitchell SE (2011) A robust, simple genotyping-bysequencing (GBS) approach for high diversity species. PLoS One 6(5):e19379

Felsenstein J (1985) Confidence limits on phylogenies: an approach using the bootstrap. Evolution 39:783-791

Ferriol M, Pico B, Nuez F (2003) Genetic diversity of a germplasm collection of Cucurbita pepo using SRAP and AFLP markers. Theor Appl Genet 107(2):271-282

Girke A, Schierholt A, Becker HC (2012) Extending the rapeseed genepool with resynthesized Brassica napus L. I: genetic diversity. Genet Resour Crop Ev 59(7):1441-1447

Glaubitz JC, Casstevens TM, Lu F, Harriman J, Elshire RJ, Sun Q, Buckler ES (2014) TASSEL-GBS: a high capacity genotyping by sequencing analysis pipeline. PLoS One 9(2):e90346

Gourret JP, Delourme R, Renard M (1992) Expression of ogu cytoplasmic male sterility in cybrids of Brassica napus. Theor Appl Genet 83(5):549-556

Grant I, Beversdorf WD (1985) Heterosis and combining ability estimates in spring-planted oilseed rape (Brassica napus L.). Can J Genet Cytol 27(4):472-478

Heyn FW (1976) Transfer of restorer genes from Raphanus to cytoplasmic male sterile Brassica napus. Cruciferae News 1: $15-16$

Jain A, Bhatia S, Banga SS, Prakash S, Lakshmikumaran M (1994) Potential use of random amplified polymorphic DNA (RAPD) technique to study the genetic diversity in Indian mustard (Brassica juncea) and its relationship to heterosis. Theor Appl Genet 88(1):116-122

Jesske T, Olberg B, Schierholt A, Becker HC (2013) Resynthesized lines from domesticated and wild Brassica taxa and their hybrids with $B$. napus L.: genetic diversity and hybrid yield. Theor Appl Genet 126(4):1053-1065

Kearse M, Moir R, Wilson A, Stones-Havas S, Cheung M, Sturrock S, Buxton S, Cooper A, Markowitz S, Duran C, Thierer T, Ashton B, Mentjies P, Drummond A (2012) Geneious basic: an integrated and extendable desktop software platform for the organization and analysis of sequence data. Bioinformatics 28(12):1647-1649

Li H, Durbin R (2010) Fast and accurate long-read alignment with burrows-wheeler transform. Bioinformatics 26(5):589-595

Li H, Homer N (2010) A survey of sequence alignment algorithms for next-generation sequencing. Brief Bioinform 11(5):473-483
Li G, Quiros CF (2001) Sequence-related amplified polymorphism (SRAP), a new marker system based on a simple PCR reaction: its application to mapping and gene tagging in brassica. Theor Appl Genet 103(2-3):455-461

Lin L, Allemekinders H, Dansby A, Campbell L, Durance-Tod S, Berger A, Jones PJ (2013) Evidence of health benefits of canola oil. Nutr Rev 71(6):370-385

Liu H, Bayer M, Druka A, Russell JR, Hackett CA, Poland J, Ramsay L, Hedley PE, Waugh R (2014) An evaluation of genotyping by sequencing (GBS) to map the Breviaristatume (ari-e) locus in cultivated barley. BMC genomics 15(1): 104

Liu K, Muse SV (2005) PowerMarker: an integrated analysis environment for genetic marker analysis. Bioinformatics 21(9):2128-2129

Lombard V, Baril CP, Dubreuil P, Blouet F, Zhang D (2000) Genetic relationships and fingerprinting of rapeseed cultivars by AFLP: consequences for varietal registration. Crop Sci 40(5):1417-1425

Lu Y, Yan J, Guimaraes CT, Taba S, Hao Z, Gao S, Xu Y (2009) Molecular characterization of global maize breeding germplasm based on genome-wide single nucleotide polymorphisms. Theor Appl Genet 120(1):93-115

McVetty PBE, Duncan RW (2014) Canola, rapeseed, and mustard: for biofuels and Bioproducts. In: Cruz V, Dierig D (eds) Industrial Crops. Springer, New York, pp. 133-156

McVetty PBE, Rimmer SR, Scarth R, Berg CVD (1996a) Neptune high erucic acid, low glucosinolate summer rape. Can J Plant Sci 76(2):343-344

McVetty PBE, Scarth R, Rimmer SR, Berg CVD (1996b) Venus high erucic acid, low glucosinolate summer rape. Can J Plant Sci 76(2):341-342

McVetty PBE, Rimmer SR, Scarth R (1998) Castor high erucic acid, low glucosinolate summer rape. Can J Plant Sci 78(2): 305-306

McVetty PBE, Fernando WGD, Scarth R, Li G (2006) Red River 1852 roundup ready ${ }^{\mathrm{TM}}$ high erucic acid, low glucosinolate summer rape. Can J Plant Sci 86(4):1181-1182

Mouchet M, Guilhaumon F, Villéger S, Mason NW, Tomasini JA, Mouillot D (2008) Towards a consensus for calculating dendrogram-based functional diversity indices. Oikos 117(5):794-800

Nei M (1972) Genetic distance between populations. American naturalist, $\mathrm{p}: 283-292$

Nei M (1987) Molecular evolutionary genetics. Columbia University Press, New York, pp. 176-253

Nielsen R, Paul JS, Albrechtsen A, Song YS (2011) Genotype and SNP calling from next-generation sequencing data. Nat Rev Genet 12(6):443-451

Nye TM, Lio P, Gilks WR (2006) A novel algorithm and webbased tool for comparing two alternative phylogenetic trees. Bioinformatics 22(1):117-119

Odong TL, van Heerwaarden J, Jansen J, van Hintum TJ, van Eeuwijk FA (2011) Determination of genetic structure of germplasm collections: are traditional hierarchical clustering methods appropriate for molecular marker data? Theor Appl Genet 123(2):195-205

Riaz A, Quiros CF (2011) Inter- and intra-cluster heterosis in spring type oilseed rape (Brassica napus L.) hybrids and prediction of heterosis using SRAP molecular markers. SABRAO J Breed Genet 43(1):27-43 
Radoev M, Becker HC, Ecke W (2008) Genetic analysis of heterosis for yield and yield components in rapeseed (Brassica napus L.) by quantitative trait locus mapping. Genetics 179(3):1547-1558

Rahman H (2013) Review: breeding spring canola (Brassica napus L.) by the use of exotic germplasm. Can J Plant Sci 93(3):363-373

Reynolds J, Weir BS, Cockerham CC (1983) Estimation of the coancestry coefficient: basis for a short-term genetic distance. Genetics 105:767-779

Riaz A, Li G, Quresh Z, Swati MS, Quiros CF (2001) Genetic diversity of oilseed Brassica napus inbred lines based on sequence-related amplified polymorphism and its relation to hybrid performance. Plant Breed 120(5):411-415

Ruiz JJ, García-Martínez S, Picó B, Gao M, Quiros CF (2005) Genetic variability and relationship of closely related Spanish traditional cultivars of tomato as detected by SRAP and SSR markers. J Am Soc Hortic Sci 130(1):88-94

Saitou N, Nei M (1987) The neighbor-joining method: a new method for reconstructing phylogenetic trees. Mol Boil Evol 4(4):406-425

Scarth R, McVetty PBE, Rimmer SR, Stefansson BR (1991) Hero summer rape. Can J Plant Sci 71(3):865-866

Scarth R, McVetty PBE, Rimmer SR (1995) Mercury high erucic low glucosinolate summer rape. Can J Plant Sci 75(1):205-206

Shull GH (1908) The composition of a field of maize. Am Breeders Assoc Rep 4:296-301

Sokal R, Michener C (1958) A statistical method for evaluating systematic relationships. University of Kansas Science Bulletin 38:1409-1438

Spindel J, Wright M, Chen C, Cobb J, Gage J, Harrington S, McCouch S (2013) Bridging the genotyping gap: using genotyping by sequencing (GBS) to add high-density SNP markers and new value to traditional bi-parental mapping and breeding populations. Theor Appl Genet 126(11):2699-2716

Sun Z, Wang Z, Tu J, Zhang J, Yu F, McVetty PB, Li G (2007) An ultradense genetic recombination map for Brassica napus, consisting of 13551 SRAP markers. Theor Appl Genet 114(8):1305-1317

Tamura K, Nei M (1993) Estimation of the number of nucleotide substitutions in the control region of mitochondrial DNA in humans and chimpanzees. Mol Biol Evol 10(3):512-526

Tamura K, Stecher G, Peterson D, Filipski A, Kumar S (2013) MEGA6: molecular evolutionary genetics analysis version 6.0. Mol Biol Evol 30(12):2725-2729

Ward JH Jr (1963) Hierarchical grouping to optimize an objective function. J Am Stat Assoc 58(301):236-244

Wen YC, Wang HZ, Shen JX, Liu GH, Zhang SF (2006) Analysis of genetic diversity and genetic basis of Chinese rapeseed cultivars (Brassica napus L.) by sequence-related amplified polymorphism markers. Sci Agric Sinica 39(2):246-256

Wittkop B, Snowdon RJ, Friedt W (2009) Status and perspectives of breeding for enhanced yield and quality of oilseed crops for Europe. Euphytica 170(1-2):131-140

Yu CY, Hu SW, Zhao HX, Guo AG, Sun GL (2005) Genetic distances revealed by morphological characters, isozymes, proteins and RAPD markers and their relationships with hybrid performance in oilseed rape (Brassica napus L.). Theor Appl Genet 110(3):511-518

Zharkikh A, Li WH (1992) Statistical properties of bootstrap estimation of phylogenetic variability from nucleotide sequences. I. Four taxa with a molecular clock. Mol Biol Evol 9(6):1119-1147 